\title{
Resistance of Feedback Amplifiers: A Novel Representation
}

\author{
Arnaldo D'amico, Member, IEEE, Christian Falconi, Member, IEEE, Gianluca Giustolisi, Member, IEEE, and \\ Gaetano Palumbo, Fellow, IEEE
}

\begin{abstract}
We propose a representation and a related methodology for evaluating the exact input and output resistances of feedback amplifiers. Our approach is based on the generalization of the Rosenstark theorem. Indeed, it requires the computation of two resistances (direct and asymptotic) each one evaluated in one of the two ideal and extreme conditions of the return ratio (zero or infinity). Due to these characteristics, the representation allows one to understand what happens to a feedback amplifier resistance in the case of absence of feedback or in the ideal case of infinite feedback.
\end{abstract}

Index Terms-Circuit analysis, circuit modeling, circuit theory, feedback amplifiers, feedback circuits.

\section{INTRODUCTION}

$\mathbf{F}$ EEDBACK has the property to change (increase or decrease) the input/output resistances (impedances) of openloop amplifiers [1]-[11]. And this property is widely used in the design of electronic and microelectronics circuits [12]-[14].

As in the case of the evaluation of feedback amplifier transfer functions, there are different methods for calculating the exact (such as [5] and [15], [16]), or approximated (as described in [3], [4] and [11]) input/output resistances of closed-loop amplifiers. Among the approaches that lead to exact results, the most immediate one is analyzing the circuit directly by writing the Kirchhoff equations of the small-signal circuit. However, this approach is computationally tedious, and does not allow one to understand deeply how the feedback modifies the properties of the original open-loop circuit.

Another exact approach models both the amplifier and the feedback network with their proper two-port models [3]. However, due to the non-unidirectional nature of real circuits, the two-port network which models each block has to be chosen judiciously and, for exact results, the analysis becomes extremely elaborate.

An interesting method to evaluate exact input and output resistances of feedback amplifier was reported in [15] by Cherry. This exact method is an extension of an approximated approach previously presented in [2]. However, the method needs to identify the feedback network, classify the feedback amplifier in one

Manuscript received July 3, 2006; revised September 18, 2006. This paper was recommended by Associate Editor A. I. Karsiliyan.

A. D'amico and C. Falconi are with the Dipartimento di Ingegneria Elettronica, Università Roma Tor Vergata, Rome 0133, Italy, and also with Instituto di Acustica O. M. Corbino, Rome 0133, Italy (e-mail falconi@eln.uniroma2.it). G. Giustolisi and G. Palumbo are with Dipartimento di Ingegneria Elettrica Elettronica e dei Sistemi (DIEES), Universita' Di Catania, I-95125 Catania, Italy, ( e-mail: ggiusto@diees.unict.it; gpalumbo@diees.unict.it,).

Digital Object Identifier 10.1109/TCSII.2006.889713 of the four classical topologies [3], and properly break the loop to evaluate the loop gain. Thus, this methodology needs an $a$ priori knowledge and a circuit expertise.

An efficient approach for evaluating exact input or output resistances of feedback amplifiers was introduced by Blackman in 1943 [16]. Subsequently, the technique, also included in the textbooks [1] and [3], was revisited by Rosenstark [17], and was developed independently by Choma through signal flow analysis [18]. Hence, the Blackman theorem represents a relevant methodology for evaluating input and output resistances of feedback amplifiers even for those who make use of the Rosenstark or the Choma method.

It is worth noting that, to the best of the authors' knowledge, while the analytical representation of the Choma method (which applies for the determination of closed-loop transfer functions) is a clear generalization of the Blackman theorem, a Rosenstark-like representation of resistances of feedback amplifiers has never been proposed. Indeed, even Rosenstark in its original manuscript suggested the use of the Blackman theorem to evaluate input and output resistances of feedback amplifiers [17]. However, as we shall discuss in the next section, despite its advantages that justify its remarkable fame, the Blackman theorem has some drawbacks, too.

In this brief, we propose an alternative representation and a related methodology for evaluating the exact input and output resistances of feedback amplifiers. The proposed approach comes out from a generalization of the Rosenstark representation, and provides some interesting features.

\section{BLACKMAN THEOREM}

Without any loss of generality, let us consider the block schema of the amplifier in Fig. 1 and assume we want to evaluate the resistance $r_{x}$ at gate a-b. Naming the amplifier return ratio as $T\left(R_{x}\right)$, where the dependence on the external resistor $R_{x}$ is explicitly shown, the Blackman theorem states that $[8]-[10]$

$$
r_{x}=r_{x, o l} \frac{1+T(0)}{1+T(\infty)} .
$$

In relationship (1), $r_{x, o l}$ is the resistance at gate a-b in openloop configuration (more in general, when the return ratio is zero); $T(0)$ and $T(\infty)$ are the return ratios evaluated when gate a-b is short-circuited $\left(R_{x}=0\right)$ or left open $\left(R_{x} \rightarrow \infty\right)$, respectively.

A drawback of the Blackman representation is due to the fact that, differently from the Rosenstark representation for the amplifier transfer function, it does not give any explicit information 


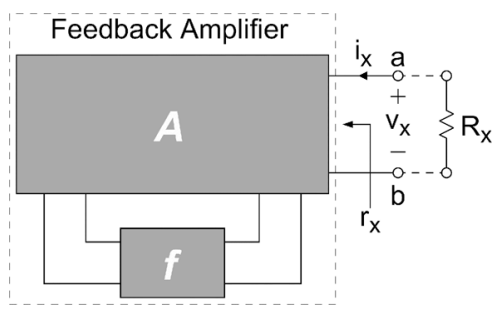

Fig. 1. Block schema of a feedback amplifier.

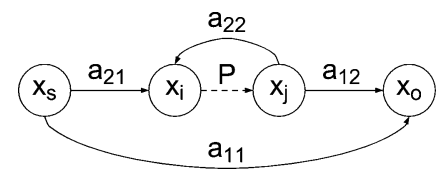

Fig. 2. General signal flow graph of a feedback circuit.

about the ideal and asymptotic value of $r_{x}$, (i.e., when $T\left(R_{x}\right) \rightarrow$ $\infty)$, which becomes an important and practical parameter in many applications. Moreover, the Blackman theorem requires two different return ratios. Thus, even if $T(0)$ and $T(\infty)$ can be evaluated from a more general relationship $T\left(R_{x}\right)$, the evaluation of $r_{x}$ is a tedious task.

\section{PRoposed RePresentation AND Methodology}

\section{A. Introduction to Flow Graph Analysis}

Consider the linear circuit represented by the signal flow graph shown in Fig. 2 [8], [11]. Variables $x_{s}$ and $x_{o}$ represent the input and output signals, moreover, two other generic variables, $x_{i}$ and $x_{j}$, linked together through the control (or critical) parameter $P$, are explicitly shown. Parameters $a_{i j}$ are the weight branches. Variables $x_{i}, x_{j}$ and the control parameter, $P$, model a controlled generator, or the relation between voltage and current across two nodes of the circuit.

Inspecting Fig. 2, we note that term $a_{11}$ is the transfer function between the input and the output, setting the control parameter, $P$, to zero; term $a_{12}$ is the transfer function between the output and the controlled variable, $x_{j}$, setting the input source, $x_{s}$, to zero; term $a_{21}$ represents the transfer function between the source variable and the inner variable, $x_{i}$, when the controlled variable, $x_{j}$, is set to zero (i.e., when the control parameter, $P$, is set to zero); term $a_{22}$ gives the relation between the independent and the controlled inner variables setting control parameter, $P$, and input variable, $x_{s}$, to zero.

Writing the variables $x_{o}$ and $x_{i}$ in terms of $x_{s}$ and $x_{j}$, we get

$$
\begin{aligned}
x_{0} & =a_{11} x_{s}+a_{12} x_{j} \\
x_{i} & =a_{21} x_{s}+a_{22} x_{j} .
\end{aligned}
$$

Then, exploiting the relationship $x_{j}=P x_{i}$ and solving (2b) for $x_{i}$, yields

$$
x_{i}=\frac{a_{21}}{1-P a_{22}} x_{s}
$$

By substituting (3) into (2a), the following relationship holds:

$$
X_{F}=\frac{x_{o}}{x_{s}}=a_{11} \frac{1}{1-P a_{22}}+\left(a_{11}-\frac{a_{12} a_{21}}{a_{22}}\right) \frac{-P a_{22}}{1-P a_{22}} .
$$

\section{B. Proposed Resistance Representation}

Relationship (4) has general validity and we can use it to evaluate the resistance $r_{x}$ at gate a-b of Fig. 1, by substituting $x_{o}$ and $x_{s}$ with voltage $v_{x}$ and current $i_{x}$, respectively. In particular, referring to gate $a-b$, relationship (4) allows us to define the direct resistance, $r_{x, o l}$, (or equivalently the resistance in open-loop configuration) and the asymptotic resistance, $r_{x, \infty}$, as those resistances evaluated when the control parameter is set to zero and infinity, respectively. Thus, we get

$$
\begin{aligned}
& r_{x, o l}=\left.\frac{v_{x}}{i_{x}}\right|_{P=0}=a_{11} \\
& r_{x, \infty}=\left.\frac{v_{x}}{i_{x}}\right|_{P \rightarrow \infty}=-\frac{a_{12} a_{21}}{a_{22}}+a_{11} .
\end{aligned}
$$

Moreover, it is apparent that once we explicitly identify the controlled source element (represented by the controlled source quantity, $x_{j}$, the controlling quantity, $x_{i}$, and the critical parameter $P$, so that $x_{j}=P x_{i}$ ), the term $a_{22} P$ is the corresponding return ratio. It is worth noting, however, that for the return ratio, the input signal ( $x_{s}$ in Fig. 2$)$ must be set to zero. This corresponds to setting the input source, $i_{x}$, to zero. Thus, the return ratio must be evaluated with the gate a-b open, that is

$$
T(\infty)=-\left.P \frac{v_{x}}{i_{x}}\right|_{i_{x}=0}=-\left.P \frac{v_{x}}{i_{x}}\right|_{R_{x} \rightarrow \infty}=-P a_{22} .
$$

In conclusion, the resistance $r_{x}$ at gate a-b takes the form

$$
r_{x}=r_{x, \infty} \frac{T(\infty)}{1+T(\infty)}+r_{x, o l} \frac{1}{1+T(\infty)}
$$

Differently from the Blackman theorem, the novel representation in (6) requires the evaluation of one return ratio only. Moreover, likewise the Rosenstark theorem, it makes use of an asymptotic term, given by resistance $r_{x, \infty}$, which describes the closed-loop resistance at gate $\mathrm{a}-\mathrm{b}$ when an ideal and infinite return ratio is assumed. Hence, even if the number of terms involved equals those of the Blackman theorem, the proposed representation introduces a new asymptotic term that has a clear physical meaning and can be more useful to the designer.

\section{Resistance Evaluation}

To evaluate the three terms of the closed-loop resistance relationship (4), we have to explicitly identify a critical controlled source (represented by $x_{j}=P x_{i}$ ) and follow the steps given below.

Step 1) Evaluation of the open-loop resistance $r_{x, o l}$ : Turn off the critical controlled source setting $P=0$ and evaluate the resistance of the gate $a-b$ to achieve its open-loop value. Note that, if $P$ is associated with a 


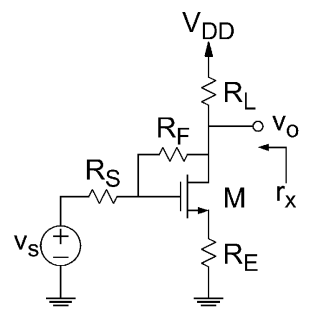

(a)

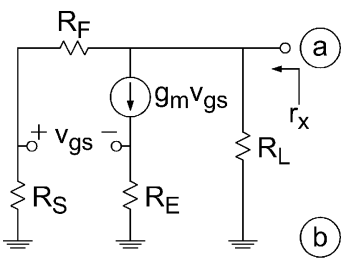

(b)

Fig. 3. Feedback amplifier example. Small-signal model.

(a) Transistor level model. (b) controlled current (voltage) source, condition $P=0$ requires an open circuit (short circuit) to replace the branch containing the controlled source.

Step 2) Evaluation of the asymptotic resistance $r_{x, \infty}$ : Set the critical parameter to infinity $(P \rightarrow \infty)$ and evaluate the resistance of the gate a-b to achieve its asymptotic value. Under this condition, since the controlled variable, $x_{j}$, must be a finite quantity, the controlling variable, $x_{i}$, is forced to 0 . Note that the asymptotic resistance, stands for the resistance under the ideal condition of infinite loop-gain.

Step 3) Evaluation of the return ratio $T(\infty)$ : Maintain the gate a-b open $\left(R_{a b} \rightarrow \infty\right)$ and replace the critical controlled voltage (current) source with an independent voltage (current) generator, $S$. Then evaluate $a_{22}=x_{i} / S$ and the return ratio as $T(\infty)=$ $-P a_{22}=-P\left(x_{i} / S\right)$.

\section{EXAMPLE}

In order to show the use of relationship (6), consider the circuit in Fig. 3(a), where a common source topology with source degeneration and a further feedback resistance, $R_{F}$, is depicted. Its small-signal model, neglecting MOS output resistance and setting to zero (i.e., shorting) the input signal generator, is plotted in Fig. 3(b).

Assume the voltage-controlled current source as the critical element and let the transconductance $g_{m}$ be the control parameter, $P$. According to Section III, setting $P=0$ and inspecting the circuit in Fig. 3(b), it is easy to find the resistance at gate a-b which results

$$
r_{x, o l}=\left(R_{S}+R_{F}\right) \| R_{L}
$$

To evaluate the asymptotic resistance, $r_{x, \infty}$, we first analyze the circuit in Fig. 4 , where also the current probe, $i_{\text {probe }}$, is included, and then we add the contribution of the resistance $R_{L}$. In the process, we consider the critical parameter infinitely large and, since the controlled variable, $g_{m} v_{g s}$, must be a finite

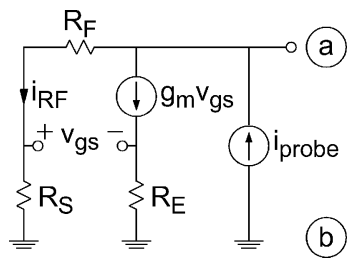

Fig. 4. Simplified circuit to evaluate the asymptotic resistance.

quantity, this means setting the voltage $v_{g s}$ equal to zero. Thus, having $v_{g}=v_{s}$, we can write

$$
i_{\mathrm{RF}}=\frac{R_{E}}{R_{S}} g_{m} v_{g s}
$$

and, considering that $i_{\text {probe }}=i_{\mathrm{RF}}+g_{m} v_{g s}$, we have

$$
g_{m} v_{g s}=\frac{R_{S}}{R_{E}+R_{S}} i_{\text {probe }}
$$

Therefore, the voltage $v_{a b}$ at gate $\mathrm{a}-\mathrm{b}$ is

$$
v_{a b}=\left(R_{F}+R_{S}\right) i_{\mathrm{RF}}=\frac{R_{F}+R_{S}}{R_{E}+R_{S}} R_{E} i_{\text {probe }}
$$

which gives

$$
r_{x, \infty, \text { simpl }}=\frac{v_{a b}}{i_{\text {probe }}}=\frac{R_{F}+R_{S}}{R_{E}+R_{S}} R_{E}
$$

and, considering the contribution of $R_{L}$, the final asymptotic resistance results

$$
r_{x, \infty}=\left(\frac{R_{F}+R_{S}}{R_{E}+R_{S}} R_{E}\right) \| R_{L}
$$

It is worth noting that (12) returns the ideal zero output resistance when a pure shunt connection is present (i.e., $R_{E}=0$ ) and returns $R_{L}$ in the case of a pure series connection (i.e., $\left.R_{F} \rightarrow \infty\right)$.

To evaluate the return ratio $T(\infty)$ we first substitute the controlled source $g_{m} v_{g s}$ with an independent current source, $i_{S}$, and then we search for the former controlling voltage, $v_{g s}$. Voltage $v_{g}$ is given by the amount of current $i_{S}$ that flows on $R_{S}$ due to the parallel between $R_{S}+R_{F}$ and $R_{L}$, that is

$$
v_{g}=-\frac{R_{S} R_{L}}{R_{F}+R_{S}+R_{L}} i_{S}
$$

whereas voltage $v_{s}$ is simply given by the product $R_{E} i_{S}$.

Therefore, we have

$T(\infty)=-\left.g_{m} \frac{v_{g s}}{i_{S}}\right|_{i_{x}=0}=g_{m}\left(\frac{R_{S} R_{L}}{R_{S}+R_{F}+R_{L}}+R_{E}\right)$.

Finally, using relationships (7), (12), and (14) into (6), we find resistance $r_{x}$, as shown in (15) at the bottom of the next page. 


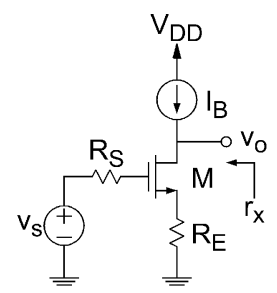

(a)

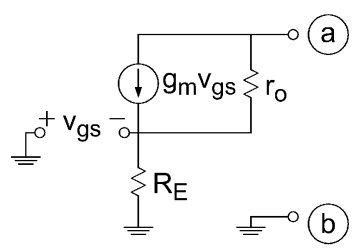

(b)

Fig. 5. Indefinite form example. (a) Transistor level model. (b) Small-signal model.

\section{REMARKS}

\section{A. Cases With Indefinite Form}

The proposed approach seems to be very interesting, since it overcomes the above mentioned drawbacks of the Blackman theorem. However, in some simple cases a difficulty could arise for the determination of an indefinite mathematical form.

For example consider the circuit shown in Fig. 5(a) and suppose we want to calculate the output resistance following the procedure discussed in Section III. The equivalent small-signal model is shown in Fig. 5(b), where also the MOS output resistance is included. The direct resistance can be immediately derived

$$
r_{x, o l}=r_{o}+R_{E}
$$

To evaluate the return ratio we note that with the gate $a-b$ open, the current through resistance $R_{E}$ is zero thus setting voltage $v_{g s}$ to zero, also. Hence, we have

$$
T(\infty)=0
$$

Regarding the asymptotic resistance, if $g_{m}$ goes to infinity and the product $g_{m} v_{g s}$ must remain a finite quantity, then $v_{g s}=$ 0 . As a consequence the current through $R_{E}$, or equivalently the probe current, is forced to be zero. Thus, we get

$$
r_{x, \infty}=\infty
$$

Substituting (16) into (6), the indefinite form " $0 \cdot \infty$ " is apparent.

The situation analyzed is common for some circuits which present a series-feedback connection at the gate of interest. In particular, it occurs when there is no shunt resistance between the two nodes of the gate.

The drawback can be simply overcome by introducing a dummy resistor between nodes a and $\mathrm{b}, R_{a b}$. Then, we determine the terms $r_{x, o l}\left(R_{a b}\right), r_{x, \infty}\left(R_{a b}\right)$ and $T\left(R_{a b}\right)$, and, after evaluating $r_{x}\left(R_{a b}\right)$ through (6), we have only to calculate the limit for $R_{a b} \rightarrow \infty$. Referring to the example in Fig. 5, under this assumption (16) becomes

$$
\begin{aligned}
r_{x, o l}\left(R_{a b}\right) & =\frac{\left(r_{o}+R_{E}\right) R_{a b}}{r_{o}+R_{E}+R_{a b}} \\
r_{x, \infty}\left(R_{a b}\right) & =R_{a b} \\
T\left(R_{a b}\right) & =\frac{g_{m} r_{o} R_{E}}{r_{o}+R_{E}+R_{a b}} .
\end{aligned}
$$

Then (6) results in (18), shown at the bottom of the page, where the indefinite form is no more present.

\section{B. Admittance Complementary Form}

Instead of the resistance, we can evaluate the admittance, $g_{x}$, at gate $a-b$ of a feedback circuit through the complementary form of relationship (6). Specifically, we can evaluate (4) for the particular case in which $x_{o}$ and $x_{s}$ are the current, $i_{x}$, and the voltage, $v_{x}$, at gate a-b, respectively. Therefore, conductance $g_{x}$ takes the form

$$
g_{x}=g_{x, o l} \frac{1}{1+T(0)}+g_{x, \infty} \frac{T(0)}{1+T(0)}
$$

$$
r_{x}=\frac{\left[\left(\frac{R_{F}+R_{S}}{R_{E}+R_{S}} R_{E}\right) \| R_{L}\right] g_{m}\left(\frac{R_{S} R_{L}}{R_{S}+R_{F}+R_{L}}+R_{E}\right)+\left(R_{F}+R_{S}\right) \| R_{L}}{1+g_{m}\left(\frac{R_{S} R_{L}}{R_{S}+R_{F}+R_{L}}+R_{E}\right)}
$$

$$
r_{x}=\lim _{R_{a b} \rightarrow \infty}\left[R_{a b} \frac{\frac{g_{m} r_{o} R_{E}}{r_{o}+R_{E}+R_{a b}}}{1+\frac{g_{m} r_{o} R_{E}}{r_{o}+R_{E}+R_{a b}}}+\frac{\left(r_{o}+R_{E}\right) R_{a b}}{r_{o}+R_{E}+R_{a b}} \frac{1}{1+\frac{g_{m} r_{o} R_{E}}{r_{o}+R_{E}+R_{a b}}}\right]=r_{o}+R_{E}+g_{m} r_{o} R_{E}
$$


In this case, once we explicitly identify the controlled source element, $g_{x, o l}$ and $g_{x, \infty}$ are evaluated as

$$
\begin{aligned}
g_{x, o l} & =\left.\frac{i_{x}}{v_{x}}\right|_{P=0} \\
g_{x, \infty} & =\left.\frac{i_{x}}{v_{x}}\right|_{P \rightarrow \infty} .
\end{aligned}
$$

Conductance $g_{x, o l}$ is the direct conductance, while, $g_{x, \infty}$ is the asymptotic conductance. Parameter $T(0)$ takes the general form $-P\left[x_{i} / x_{j}\right]_{x_{s}=0}$. Note, however, that in this case, setting $x_{s}=0$ means setting $v_{x}=0$. Hence, $T(0)$ is evaluated when the input source, $v_{x}$, is zero, that is when the gate a-b is shortcircuited, namely

$$
T(0)=-\left.P \frac{x_{i}}{x_{j}}\right|_{v_{x}=0}=-\left.P \frac{x_{i}}{x_{j}}\right|_{R_{x}=0} .
$$

It is worth noting that the use of the complementary form to calculate the admittance of a feedback circuit could represent an alternative way to solve the cases in which relationship (6) has an indefinite form.

\section{Resulting Properties}

Once the controlled source element is explicitly defined, a comparison between the definitions of (5a)-(5b) and (20a)-(20b) reveals that

$$
\begin{aligned}
r_{x, o l} & =\frac{1}{g_{x, o l}} \\
r_{x, \infty} & =\frac{1}{g_{x, \infty}} .
\end{aligned}
$$

Moreover, since

$$
r_{x}=\frac{1}{g_{x}}
$$

substituting (5) and (20) into (22) and taking into account (21), allows us to write

$$
\frac{r_{x, o l}+r_{x, \infty} T(\infty)}{1+T(\infty)}=\frac{r_{x, o l} r_{x, \infty}[1+T(\infty)]}{r_{x, o l} T(o)+r_{x, \infty}}
$$

which, after manipulation, leads to

$$
\frac{r_{x, o l} T(0)}{r_{x, \infty} T(\infty)}+\frac{r_{x, \infty}}{r_{x, o l}}=\frac{T(0)}{T(\infty)}+1
$$

Relationship (24) is satisfied if ${ }^{1}$

$$
\frac{T(\infty)}{T(0)}=\frac{r_{x, o l}}{r_{x, \infty}}=\frac{g_{x, \infty}}{g_{x, o l}}
$$

which relates the four quantity discussed in the present brief.

\footnotetext{
${ }^{1}$ Equation (25) is satisfied for $r_{x, o l}=r_{x, \infty}$, also. However, this solution is unrealistic.
}

\section{CONCLUSION}

In this brief, we have proposed an alternative representation and a related methodology for evaluating the exact input and output resistances of feedback amplifiers. It is a generalization of the Rosenstark representation, and, differently from the Blackman approach (that concentrates on one resistance, and on two return ratio terms), our approach focuses on one return ratio, only, and on two resistances (direct and asymptotic) each one evaluated in one of the two ideal and extreme conditions of the return ratio (zero or infinity). Due to this characteristic, the representation allows one to understand what happens to a feedback amplifier resistance in the case of absence of feedback or in the ideal case of infinite feedback.

\section{REFERENCES}

[1] H. Bode, Network Analysis and Feedback Amplifier Design. New York: van Nostrand, 1945.

[2] E. Cherry and D. Hopper, Amplifying Devices and Low-Pass Amplifier Design. New York: Wiley, 1968.

[3] J. Millman and A. Grabel, Microelectronics, 2nd ed. New York: McGraw-Hill, 1987.

[4] A. Sedra and K. Smith, Microelectronic Circuits, 3rd ed. Philadelphia, PA: Saunders, 1991.

[5] P. Gray, P. Hurst, S. Lewis, and R. Meyer, Analysis and Design of Analog Integrated Circuits, 4th ed. New York: Wiley, 2001.

[6] K. Laker and W. Sansen, Design of Analog Integrated Circuits and Systems. New York: McGraw-Hill, 1994.

[7] D. Johns and K. Martin, Analog Integrated Circuit Design. New York: Wiley, 1997.

[8] G. Palumbo and J. Choma Jr., "An overview of analog feedback Part I: Basic theory," Anal. Integr. Circuits Signal Process., vol. 17, no. 3, pp. 175-194, Nov. 1998.

[9] _ "An overview of analog feedback Part II: Amplifier configurations in generic device technologies," Anal. Integr. Circuits Signal Process., vol. 17, no. 3, pp. 195-219, Nov. 1998.

[10] G. Palumbo and S. Pennisi, Feedback Amplifiers (Theory and Design). Reading, MA: Kluwer, 2002.

[11] J. Marrero, "Simplified analysis of feedback amplifiers," IEEE Trans. Educ., vol. 48, no. 1, pp. 53-59, Feb. 2005.

[12] J. Ramirez-Angulo, R. G. Carvajal, and A. Torralba, "Low supply voltage high-performance CMOS current mirror with low input and output voltage requirements," IEEE Trans. Circuits Syst. II, Exp. Briefs, vol. 51, no. 3, pp. 124-129, Mar. 2004.

[13] "A regulated body-driven CMOS current mirror for low-voltage applications," IEEE Trans. Circuits Syst. II, Exp. Briefs, vol. 51, no. 10, pp. 571-577, Oct. 2004.

[14] S. M. Potirakis and G. E. Alexakis, "An accurate calculation of Miller effect on the frequency response and on the input and output impedance of feedback amplifiers," IEEE Trans. Circuits Syst. II, Exp. Briefs, vol. 52, no. 8, pp. 491-495, Aug. 2005.

[15] E. Cherry, "Input impedance and output impedance of feedback amplifiers," IEE Proc. Circuits Devices Syst., vol. 143, no. 4, pp. 195-201, Aug. 1996.

[16] R. Blackman, "Effect of feedback on impedance," BSTJ, vol. 22, pp. 269-277, Oct. 1943.

[17] S. Rosenstark, "A simplified method of feedback amplifier analysis," IEEE Trans. Educ., vol. E-17, no. 4, pp. 192-198, Nov. 1974.

[18] J. Choma Jr., "Signal flow analysis of feedback networks," IEEE Trans. Circuits Syst., vol. 37, no. 4, pp. 455-463, Apr. 1990. 